municated to rats by inoculation with the materies morbi of a cattle-disease.

Lieutenant-Colonel Leishman has throughout taken an interest in this subject and has now joined in the investigation of this point, regarding which we hope to make a further communication. In the meantime what has been written above may lead to fruitful investigation in plague areas, especially by officers of the Veterinary Services in India.

At the same time it has to be borne in mind that the tick may be the medium by which infection is conveyed from the soil to both man and beast, as well as from beast to man.

REFERENCES

1 Indian Famine Commission, 1878, Part III, Famine History, p. 172 3 Josephus, tranolated by W. Whiston, 1863, p. 154. 8 Agricultural Ledger 3896, Special Veteriaury Series, No. 1 , p 19. 41bid, p. $\epsilon_{5}$. 5 Ibid, 1899 No. 7. The Cattle of Kuniáo o, by Veterinary Lteutenant: G. K. Walker. - Bombay Agricultural Report for ${ }_{1892-33}$ p. 22. 7 Agricultural Ledger, 899,

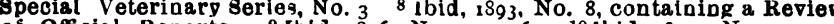

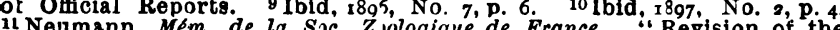
In

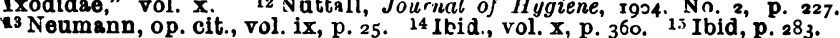

\section{AN INVESTIGATION ON THE REGENERATION OF NERVES,}

WITH RHGARD TO THE TREATMENT OF CERTAIN PARALYSES. Bx BASIL KILVINGTON, M.S., M.D.Mei B., Junior Lesturer in Anatomy, Melbourne University. (From the Physiological Laboratory.)

[PART II.]

IN a prerious communication, published in the BRITISH Medical Journal of April 29th, 1905, it was shown that the central end of one nerve is capable of establishing connexion with the distal ends of two and presumably more than two nerves. The nerves used were the external and internal popliteals which innervate antagonistic muscles. It was shown that a very use'ul function could be obtained in this way. As the result of counting the fibres in the regenerated nerves it was seen that a single axis cylinder in the central nerve split up into two or more branches at the place of section, and each of these could establish a connexion with a fibre in a distal nerve. It seems probable-and especially where a small nerve is used-that the nerve fibres do not reach the normal number, and presumably the function of the muscles cannot be quite as $\mathrm{t}$ ficient as in the healthy state. It was also evident from the axon reflex phenomenon that some of the fibres which come from the splitting of a single axis cylinder may supp'y antagonistic muscles. It is impossible to say what proportion of fibres do this, but from the very vigorous axon $r+f l e x$ obtained it seems that a considerable number have this arrangement.

When a nerve is divided and immediately sutured, the fibres of the central end are described as dividing and forming a kind of brush. These have a wavr course, and ultimately one branch from each axis cylinder joins with or grows down a fibre in the distal nerve.

The remainder of the branches from the central fibre gradually disappear. But if a more than corresponding number of fibres be present in the distal end some of these branches instead of disappearing remain permanently and have a definite use. I attach considerable importance to this splitting of the axis cylinder. Il a single motor cell is supplying antagonistic muscles, it is evident that there is a certain amount of waste of nerve and muscular energy. Accurate dissociated movements will be impossible, or at any rate only possible by ceasing to use such muscular fibres as are thus innervated. This is a very serious drawback to nerve crossing. That this is no theoretical objection I hope to show, and suggest a very simple remedy. Most of the nerve suturing has up to the present been done with the facial and spinal accessory which are quite analogous to the motor nerves of the limb. Aiter facio-spinal-accessory anastomosis any movement of the face has been associated with elevation of the shoulder and bending of the neck. owing to simultaneous contraction of the trapezius and sterno-mastoid muscles. This improves to some extent with time as the result of re-education of the cortical and other centres, but seems never quite to disappear. Ballance and Purves Stewart ${ }^{2}$ have reported 7 cases (in one the hypoglossal and facial were used) and say. "So far in our series of facio accessory cases, we have not observed any in. dependent movement of the face unassociated with that of the traperius and sternc-mastoid, although in several cases 4 minimal innervation of the trapezius is sufficient to cause facial contraction."

The following experiment in a dog gave the best functional results $I$ have seen, and single axis cylinders dividing to supply antagonistic muscles were not present.

Dog V. Left leg operated on January 17th. 1905. About one inch of the external popliteal nerve was excised The internal poplitegl was separated into two parts. The separation was made between the fasoicular bundies, and as little damage as possible was done to the nerve tissue. These were then cut transversely across, and the smaller part was sutured to the distal end of the external popliteal. The larger one was then fixed by a single suture to the distal end of the entire internal popliteal (see Fig. I). Union of the wound took place by first intention.
A

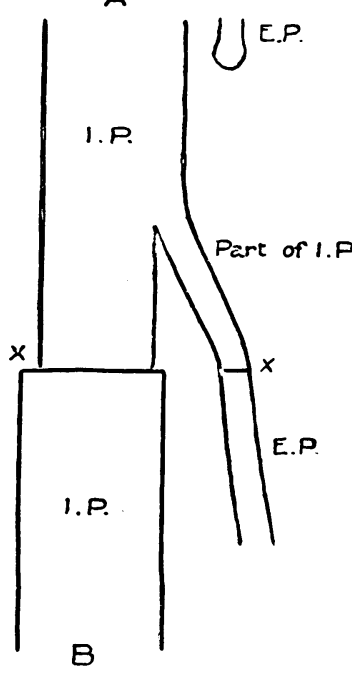

Fig. x.
A

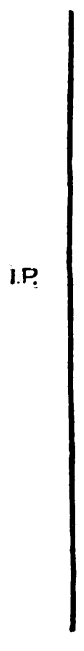

C
March 7th, r905. Dog walked fairly well and nearly always on the plantar surface of the foot; no ulcers had formed on the dursum. The original wound was opened up. Two small bulbs had formed at $x \times$ where the suturing had been done, but these were not in contact with one another.

June 7 th, 1905 (141 days). The function was practically perfect, and it was almost impossible to tell which leg had been operated on. scratching, which I regard as the best test of accurate co ordination in the dog, was done nearly perfectly. The animal was anaesthetized and electrical stimulation performed. The internal popliteal was cut across at $A$, and stimulation here produced a vigorous response in the muscles at the front and back of the leg. The internal popliteal was then divided across at $B$, and stimulation at $A$ now produced only contraction of the extensors of the toes. Stimulation at $B$ gave no contraction of the extensor muscle. This showed the axon reflex was absent, and none of the axis cylinders had split to give brancbes to antagonistic muscles. The anterior and posterior sets of muscles were now dissected out; they were well developed and nearly as bulky as on the opposite side.

Dog Vr. The left leg operated on February 17th, rgo5. The same operation was performed as on the previous dog, a little over $\frac{3}{4}$ in. of external popliteal being excised. Union took place by first intention. March roth. Animal up to the present has always kept the leg raised off the ground.

April 25th. It was notlced that the function was not quite so good as it had been a week or two previously. The animal very often walked on the dorsum of the foot and a small ulcer had formed here. The wound was reopened, and it was found that the bulbs at $x \times$ had fused considerably. The narrow popliteal space in the dog renders this difficult to aroid. The bulbs were separated by cutting between them and the wound sutured.

June 2oth. The function had greatly improved, but was not by any means perfect; the animal, however, rarely went over on the dorsum of the foot, and the ulcer here had healed.

\section{Concluajons.}

Both clinical and theoretical considerations point to nerve splitting being preferable to suturing two distal nerves directly to the central end of one of them. The functional result in Dog V was the best I have obtained up to the present. Dog VI is of special interest. Where the bulbs fused, fibrils from one axis cylinder presumably grew down into the two distal nerve trunks. The separation of the bulbs, even though it destroyed a good many fibres, resulted in a rapid and considerable improvement. In facio accessory anastomosis it would be wise to split the latter nerve into two strands, injuring the actual nerve fibres as little as possible. 
One of these should be attached to the distal cut end of the facial, and the other to the whole of its own distal end. This would abolish associated movements of the shoulder, or, at any rate, reduce it to a very small amount, for a few fibres at the angle of splitting would possibly be injured, resulting in their growing down the nerves to both sets of muscle. No very considerable atrophy of the muscles would take place atter this, as the fibres of the central end would multiply at the point of suture.

It is suggested that norve splitting should be made use of in the treatment of suitable cases of anterior poliomyelitis for the same reason.

In Part I of this paper it was suggested that nerve implantation would be of servlce where a considerable length of nerve was destroyed as the result of traumatism or other cause. It would be advisable, if possible, to make some use of the central end of the injured nerve. The following experiments were made to elucidate this point, and they show several interesting results.

Dog VII had the left leg operated on on September ${ }_{3} \mathrm{rd}$, 1904 (Fig. 2). About $x$ in. of the external popliteal was removed. The interual popliteal was cut nearly half-way through, and into the gaps the central and peripheral ends of external popliteal were fixed by single sutures of chromicized catgut. Some superficial suppuration took place but did not extend deeply, and an ulcer formed over the os calcis, which subsequently healed. ()a December $22 \mathrm{nd}$, 1904, the dog walked well, and rarely went over on the dorsum, except when standing on the hind legs, when the left fost doubled over; jumping was well performed. The leg muscles were smaller than those on the opposite side.

February 2 ist, 1905 (171 days). Dog was anaesthetlzed and the nerves stimulated. Internal popliteal and external popliteal were cut completely across at $A$ and $B$, stimulation at $A$ with the faradic current caused contraction of both flexor and extensor muscles of the toes, but the former were the more powerful. Stimulation of external popliteal at $B$ caused the same thing, but not so vigorously, and the flexor muscles still predominated. After cutting the internal popliteal at $C$, stimulation here resulted in forcible contraction of the extensor muscles; in fact this was more powerful than on stimulation of the external popliteal at $\mathrm{B}$.

In Dog VIII the same operation was performed and it was tested electrically after 128 days. Stimulation of the external popliteal at $D$ resulted in contraction of the flexor muscles of the toes. After cutting the internal popliteal nearly half-way through at $Q$ (Fig. 2), this axon reflex was lost. This shows that it was probably only those fibres which were thus cut across at the operation which had divided and sent branches to antagonistic muscles.

It is evident that the trunk of the internal popliteal between the two points where external popliteal was implanted in it, contained at least four varieties of fibres which are shown in Flg. 3, and the condition is a very complex one.

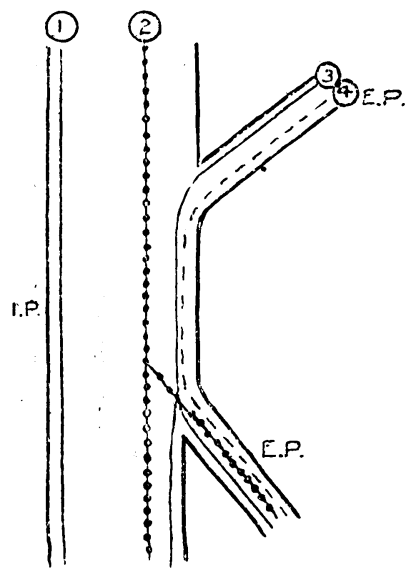

Fig. 3

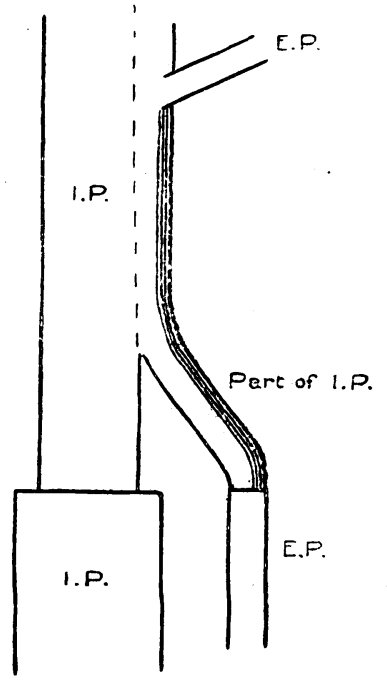

Fig. 4.
A very important point is that it is presumably a matter of indifference what length of external popliteal is excised. After loss of a short part of a nerve trunk in a limb the two ends can be brought together by stretching the nerves and relaxing the limb. An interval of not more than 2 in. at the most can be bridged over by some material which offers an absorbable scaffolding down which nerve fibres can find their way. This is supposed to act as a guide to fibres coming from the central end, but I am not aware that this has actually. been proved. It seems immaterial what absorbable substance is used for this purpose. A piece of nerve exeised from some animal apparently does no better than several strands of catgut. But none of these methods can offer the induce. ment that a degenerated nerve in situ does, for this exerts a definite chemiotactic attraction for nerve fi bres to grow down it. When this interval of about 2 in. is exceeded the resources of surgery are at present very inadequate. Probably even at this limit the functional result would be unsatisfactory. The very extensive operation of shortening the limb by resecting the $b$,ne to allow of the separated ends of the nerve being approximated is advocated, but this results in mutilation and is a serious operation. Nerve implantation in these cases is almost ignored. Cheyne and Burghard ${ }^{2}$ mention it only to condemn it.

The cause of failure seems to be due to the fact that only the sheath of the sound nerve is opened, and few, if any, of its fibres are divided. When the injured nerve is implanted in such a way it has no chance of getting any fibres to grow down its trunk. It is essential that considerable gaps dividing many of the fibres should be made in the second nerve, and into these gaps the central and peripheral ends of the injured one should be fixed. It is a dubious point as to whether one ought to use the central end of the injured nerve as in the above experiment. The hind legs of the dog do not allow one to judge accurately of the finer movements. As far as can be decided from these cases, it would be better not to use the central end in this way. Dog VII showed that the contraction resulting from stimulation of the external popliteal central to the graft was less vigorous than that resulting from the axon reflex, or in other words, the fibres actually proceeding from the central to the peripheral part of the implanted nerve produce less result than those which are coming from the sound nerve by axon splitting into the implanted nerve.

The alternative method allows of splitting of the healthy nerve and does away with the axon reflex. It can be easily seen that certain strands in a nerve occupy a particular position in the nerve trunk. For $\epsilon$ xample, the external popliteal fasciculi occupy the outer part of the sciatic trunk, and can easily be dissected for a considerable distance up the thigh. In fact, they seem to be anatomically separate up to the pelvis, and it is not uncommon to find the sciatic nerve coming out of the sciatic foramen in two trunks, the external and internal popliteal separated by some of the fibres of the pyriformis muscle. The same separation can be made for a shorter distance with the muscular twigs coming off from the nerve trunk. To apply this to the condition in question the piece of internal popliteal sutured to the distal end of the external popliteal can be separated up along the dotted line. (See Fig. 4.) If a short cut be made in this for the central end of the external popliteal to be inserted, some of the external popliteal's fibres would now reach their own distal trunk. At the same time few or none would run into the rest of the internal popliteal going to the flexor muscles, and no associated movements would result. A part of the internal popliteal which is split off plays somewhat the rôle of a graft (part shaded to the right of diagram), while the rest of the split consists of internal popliteal fibres going now by means of the external popliteal to the extensors of the toes and peroneii. The actual condition of the nerves can be seen by a glance at the diagram. This form of nerve implantation would be chiefly applicable to the arm and forearm for anatomical reasons.

I have to thank Professor Osborne for many suggestions and for his valuable help in performing the electrical stimulation.

1 Ballance and Purves Stewart. Bentcal Jodrnal, May and 1903. 2 Cheyne and Burghard, Manual of Surgical Treatment.

\section{A PROBABLE EXPLANATION OF SOME DROWNING ACCIDENTS.}

By HUGH LAWRIE, M.B., C.M.. D.P.H., Ramsbottom, Lancs.

THE following experience occurred to me on July 8th, and may throw some light upon the too frequent occurrence of drowning accidents in swimmers.

I had been cycling on the morning round, covering perhaps about five miles altogether on indifferent roads. It was a very hot day, and I was perspiring freely, though the pace was easy. While returning about I p.m. I met a friend and 